\title{
An integrated method for extracting the statistical distribution of RTN time constants
}

\author{
Mehzabeen Mehedi, Kean H. Tok, Jian F. Zhang, Zhigang Ji*, Zengliang Ye, Weidong Zhang, and \\ John S. Marsland \\ School of Engineering, Liverpool John Moores University, \\ Byrom Street, Liverpool L3 3AF, UK \\ *Also at School of Microelectronics, Shanghai Jiaotong University, Shanghai 200240, P. R. China \\ Email: j.f.zhang@ljmu.ac.uk
}

\begin{abstract}
Modelling Random Telegraph Noise (RTN) is a challenging task and its accuracy is generally unknown. The Monte Carlo modelling in the time domain requires the statistical distribution of the capture and emission time (CET) constant of traps. Although a lot of efforts were made in early works to extract the CET of individual traps, the number of traps measured is generally too limited to establish the statistical distribution of CET reliably and there are disagreements on the statistical models of CET. Two models proposed by early works are Log-normal and Log-uniform distributions, which gives very different predictions for the RTN and this difference increases as the time window becomes wider. As an accurate modelling of RTN cannot be achieved without a trustable statistical distribution of CET, it is important to find a method that allows extracting the CET distribution reliably. In contrast with early works that focus on measuring the CET of individual traps, this work proposes an integrated method for extracting the statistical distribution of CET.
\end{abstract}

\section{Introduction}

Random Telegraph Noise (RTN) is a well known phenomenon and has been investigated for many decades [1-10]. It is generally believed that RTN originates from capture-emission of charge carriers from the conduction channel of MOSFETs by individual traps [1-10]. Early works used RTN to probe individual traps and we have learnt a lot from these works. Although RTN has an adverse impact on analogue and RF circuits, its effect on digital logic circuits used to be insignificant when MOSFETs are large. This is because there are many traps in large MOSFETs, so that the average charging and discharging reach a dynamic balance. The impact of a single trap on the device is small and the channel current, Id, fluctuates little.

As the downscaling of device sizes continues and reaches nanometer scale, the impact of an individual trap on the device can be substantial. It has been reported that a single charge can reduce Id by $10 \%$, a level typically used to define device lifetime [5,11-15]. This has led to a lot of recent research in modelling RTN [2-10].

For the Monte Carlo modelling of RTN in the time domain, one needs the statistical distributions of capture and emission time (CET), RTN amplitudes, and number of traps per device. It is generally agreed that the number of traps per device follows the Poisson distribution [2,13]. Many efforts have been made to establish the statistical model for the RTN amplitude [2,8]. In contrast, there are less works on the statistical distribution of CET. Although many early works measured the CET of individual traps, the number of traps probed is typically too low to extract the statistical distribution reliably [3,6].

Two statistical models have been proposed for CET: Loguniform [1,2,7] and Log-normal [3]. Fig.1 shows that these two are very different. On one hand, the Log-normal distribution predicts that there are few traps for large time window, so that the cumulative distribution function (CDF) approaches saturation as time increases. On the other hand, the Log-uniform requires the number of traps increases linearly against logarithmic time scale and there is no saturation. The objective of this work is to develop new methodology for reliable extraction of CET distribution and then use it to assess the applicability of the statistical models proposed by early works.

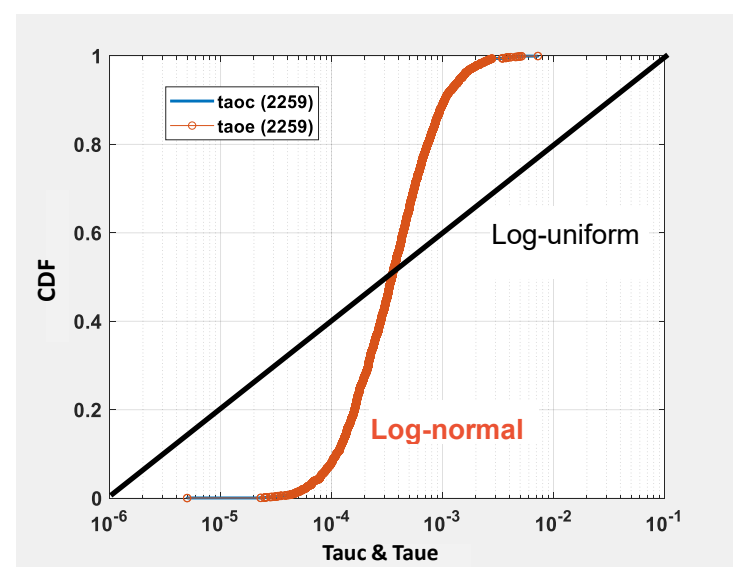

Fig. 1. A comparison of the CDF of Log-uniform and Lognormal statistical distributions. 


\section{Methodology}

To obtain the statistical distribution of CET, most of early works follow a bottom-up approach: measuring the CET of each trap and then grouping them together $[3,6]$. When there are only one or two traps in a device, their CETs can be reliably extracted. As time window increases, the number of active traps increases and it becomes difficult to extract the CET of each trap. One example is given in Fig. 2, where multiple traps in a device result in complex signals and it is a challenge to separate one trap from the rest. As a result, the bottom-up approach has its limitations and the number of CETs reported based on it is typically too low to establish a statistical distribution reliably.

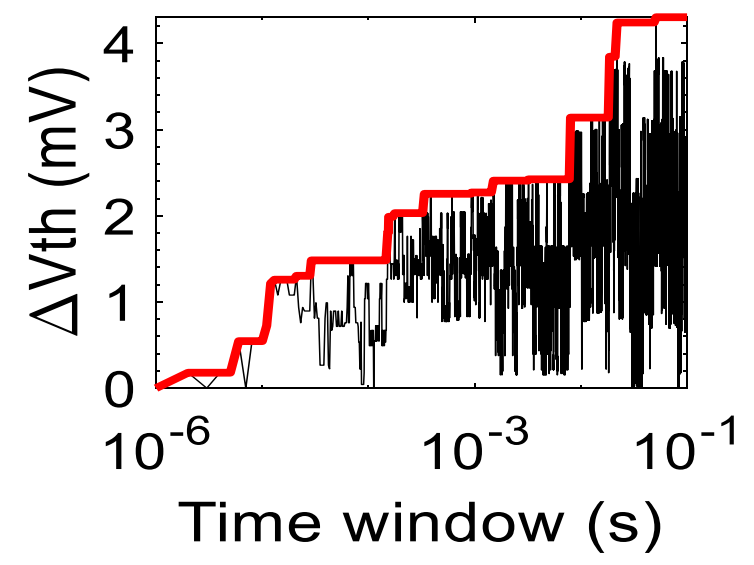

Fig. 2. An example of complex RTN signal measured in a device with multiple traps. The red lines are the envelope of the RTN signal.

In this work, we propose a new integrated methodology for extracting the statistical distribution of CETs. Instead of measuring the CET of each trap, we measure their cumulative impact on the device collectively and use it to extract the statistical distribution of CET.

The principle of this methodology is illustrated in Fig. 3, where a device has five traps with a distributed CET. The fastest trap shows up first, resulting in the first up-step, as marked out by ' (1)' in Figs. 3(a) and (b). As time increases, the charging-discharging of this trap causes many steplike changes, but the height of the step remains the same. When the second trap becomes active, its charging leads to a further increase of the envelope [16-21], as marked out by '(2)' in Fig. 3(a) and (c). As time increases further, slower traps gradually become active and each of them causes a further increase in the envelope. The increase of the envelope results from the distributed CETs, therefore. This allows extracting the statistical distribution of CETs by measuring the envelopes of multiple devices.
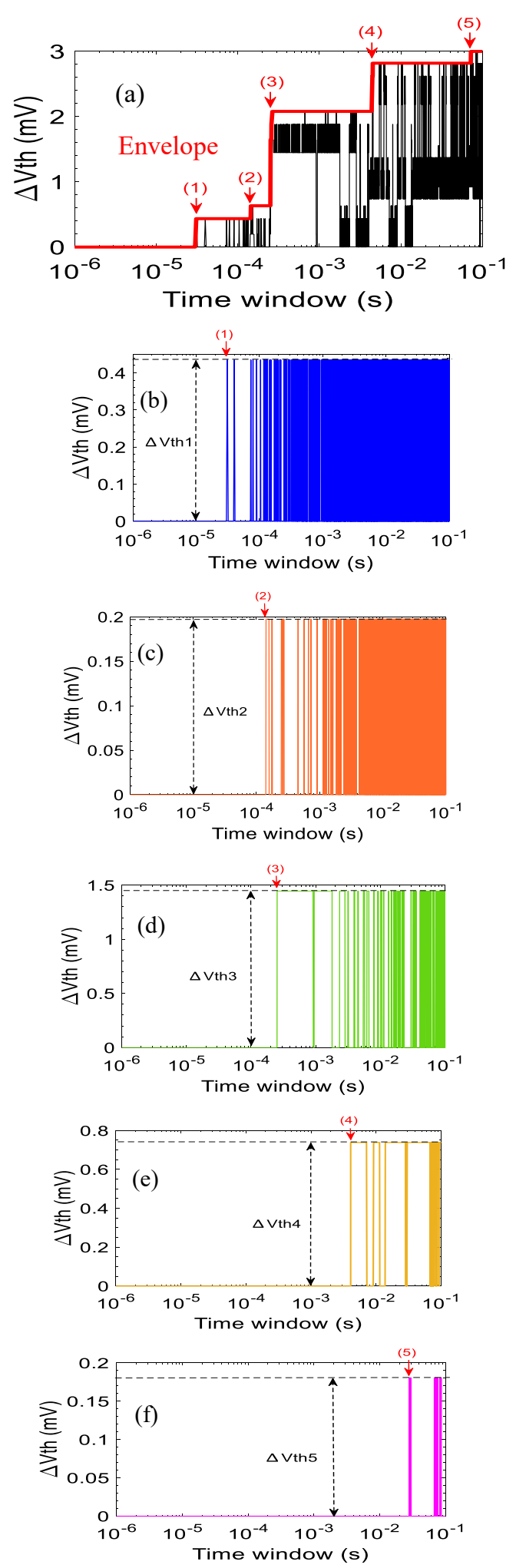

Fig. 3. An illustration of the integrated methodology. There are five traps in this device. The cumulative impact is given in (a) and their individual impacts are given in (b)-(f) [7]. 


\section{Results and discussions}

To support the proposed integrated methodology, simulation is carried out. In Figs. 4(a) and (b), we assume that the CET follows the Log-uniform and Log-normal distribution, respectively. These distributions were used to generate the CET of traps randomly. These traps were then Poisson-distributed into 400 devices. The RTN of each device is simulated and the envelope of the RTN signal is extracted as shown in Fig. 3(a). The details for extracting the envelop (Env) from the RTN signal can be found from early works [16-21]. Each gray line in Fig. 4 represents one device and the average envelope is represented by the thick black lines.
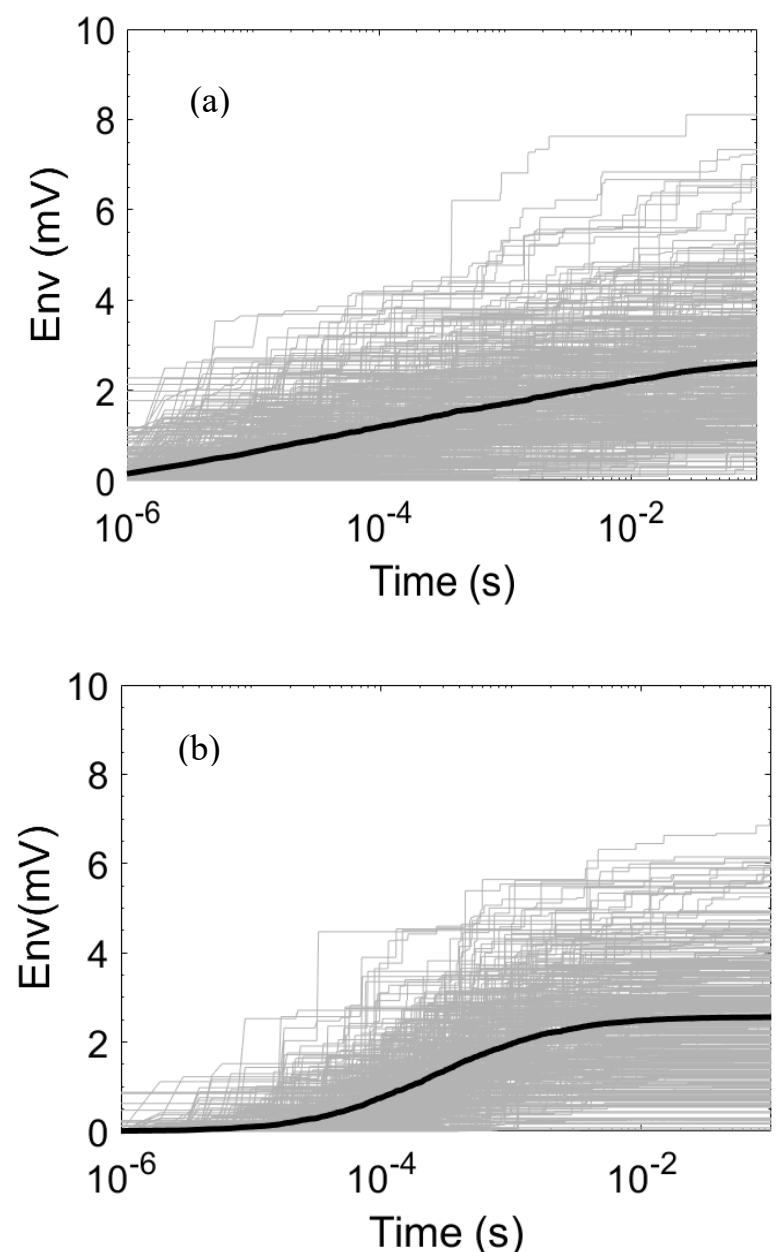

Fig. 4. The simulation of 400 devices by assuming their CET following (a) Log-uniform and (b) Log-normal distributions, respectively. The thick black lines are the average [7].

As expected, the envelope of individual device changes in steps. Their average, however, is a smooth function of time. Importantly the average faithfully reveals the underline statistics. The results confirm the proposed methodology, therefore. Since the average is an effective integration of multiple traps from many devices, this method is referred to as "integrated method".

The envelope of experimentally measured RTN is given in Fig. 5. Here we used two oscilloscopes to cover a wide time window.

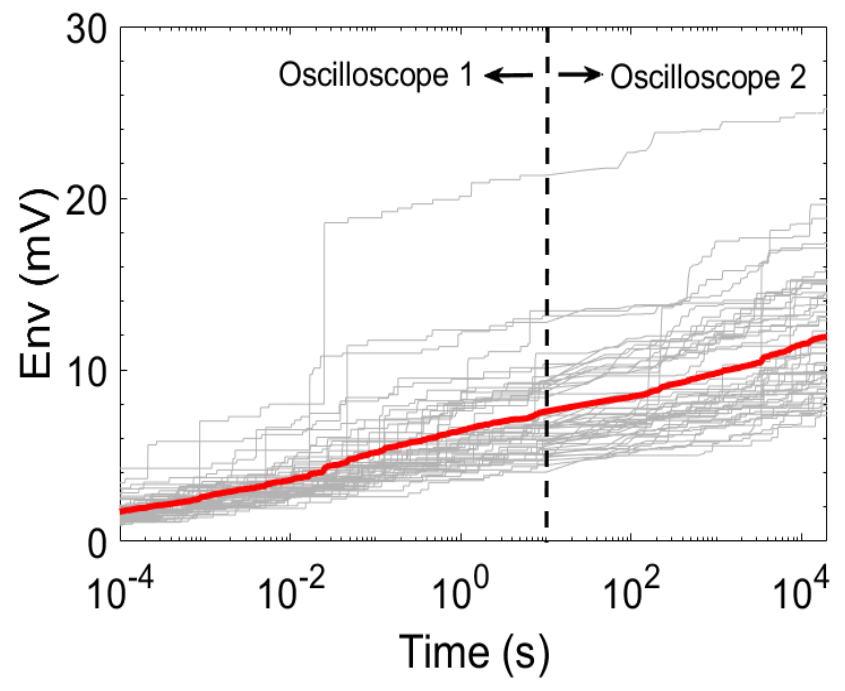

Fig. 5. The envelope of measured RTN. Each grey line is from a different device. The red line is their average [7].

The test data within $10 \mathrm{sec}$ (the blue symbols) were used to fit the Log-uniform and Log-normal distributions in Figs. 6(a) and (b), respectively. The fitted results are shown as the solid black lines. It can be seen that both distributions can be fitted well with test data. When the fitted distribution is extrapolated to longer time window, however, the dashed lines give substantially different predictions. As a result, a good fit with experimental data should not be used as the criterion to verify a model.

As mentioned earlier, a signature of the Log-uniform model is that it predicts a linear increase of the envelope with logarithmic time. This agrees well with the experimental data between 10 and $2 \times 10^{4} \mathrm{sec}$ in Fig. 6a (red symbols). As the red symbols were not used to fit the model, the good agreement between them and the model verifies the predicative capability of Log-uniform model. In contrast, the Log-normal model predicts a saturation of envelope with time, as shown by the dashed black curve in Fig. 6b. This disagrees with the test data. We conclude that the experimental data support the Log-uniform, rather than Log-normal, model.

\section{Conclusions}

This work proposes an integrated methodology for extracting the statistical distribution of capture and 
emission time constants. By measuring the cumulative impact of traps on multiple devices against time window, the experimental results are used to extract the CDF of CETs. Although the test data within a short time window can be fitted well with both Log-uniform and Log-normal distributions, only the Log-uniform model correctly predicts the long term RTN behavior.

\section{Acknowledgments}

The test samples were supplied by D. Vigar of CSR. This work is supported by EPSRC of UK under the grant no. EP/L010607/1 and EP/T026022/1.
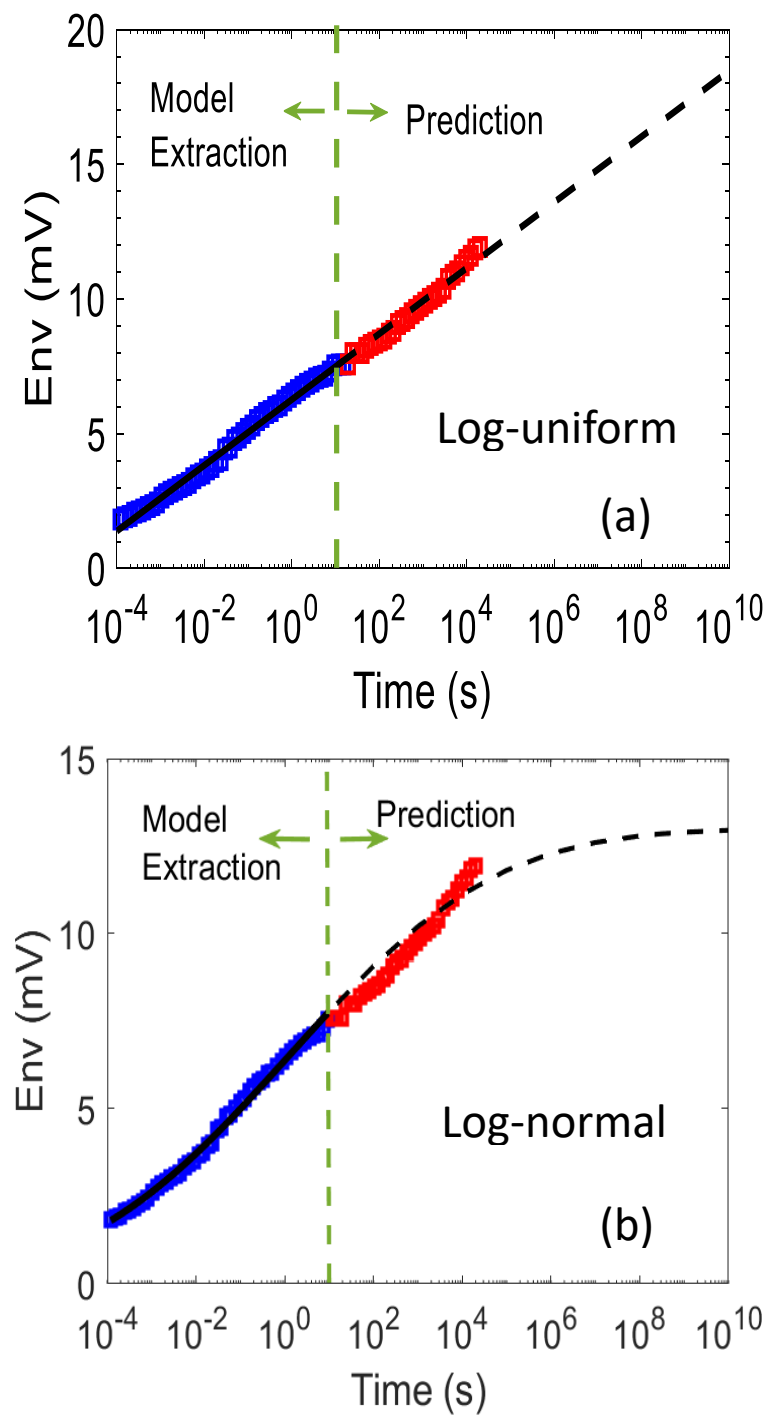

Fig. 6. The test data within $10 \mathrm{sec}$ (blue symbols) were used to fit the Log-uniform (a) and Log-normal (b) CDFs. The fitted models (Solid black lines) were then extrapolated to longer time (the dashed line/curve) and compared with the test data (red symbols) [7].

\section{References}

[1] M. J. Kirton and M. J. Uren, Advances in Physics, 38, p.367 (1989)

[2] R. Wang et al., IEDM, p.388 (2018).

[3] M. Tanizawa et al, VLSI Technol., p.95 (2010).

[4] A. Manut, R. Gao, J. F. Zhang, Z. Ji , M. Mehedi, W. D. Zhang, D. Vigar, A. Asenov, and B. Kaczer, IEEE Trans. Elec. Dev., 66, p.1482 (2019).

[5] H. Miki et al., VLSI Technol., p.148, (2011).

[6] T. Nagumo et al, IEDM, p.628, 2010.

[7] M. Mehedi, K. H. Tok, J. F. Zhang, Z. Ji, Z. Ye, W. Zhang, and J. S. Marsland, IEEE Access, 8, p.1496 (2020).

[8] M. Mehedi, K. H. Tok, Z. Ye, J. F. Zhang, Z. Ji, W. Zhang, and J. S. Marsland, IEEE Access, 9, p.43551 (2021).

[9] J. Brown, R. Gao, Z. Ji, J. Chen, J. Wu, J. F. Zhang, B. Zhou, Q. Shi, J. Crowford, and W. Zhang, VLSI Technol., p.95 (2018).

[10] J. Brown, J. F. Zhang, B. Zhou, M. Mehedi, P. Freitas, J. S. Marsland and Z. Ji, Scientific Reports, 10, (2020).

[11] J. F. Zhang, Z. Ji, and W. Zhang, Microelectronics Reliability, 80, p.109 (2018).

[12] Z. Ji, S. F. W. M. Hatta, J. F. Zhang, J. G. Ma, W. Zhang, N. Soin, B. Kaczer, S. De Gendt, and G. Groeseneken, IEDM, p.413 (2013).

[13] R. Gao, Z. Ji, A. B. Manut, J. F. Zhang, J. Franco, S. W. M. Hatta, W. D. Zhang, B. Kaczer, D. Linten, and G. Groeseneken, IEEE Trans. Elec. Dev., 64, p.4011 (2017).

[14] R. Gao, A. B. Manut, Z. Ji, J. Ma, M. Duan, J. F. Zhang, J. Franco, S. W. M. Hatta, W. Zhang, B. Kaczer, D. Vigar, D. Linten, and G. Groeseneken, IEEE Trans. Elec. Dev., 64, p.1467 (2017).

[15] M. Duan, J. F. Zhang, Z. Ji, W. Zhang, B. Kaczer, S. De Gendt, and G. Groeseneken, IEEE Elec. Dev. Lett., 33, p.480 (2012).

[16] M. Duan, J. F. Zhang, Z. Ji, W. Zhang, B. Kaczer, T. Schram, R. Ritzenthaler, G. Groeseneken, and A. Asenov, IEEE Trans. Electron Dev., 60, p.2505 (2013).

[17] M. Duan, J. F. Zhang, Z. Ji, W. D. Zhang, B. Kaczer, T. Schram, R. Ritzenthaler, G. Groeseneken, and A. Asenov, IEEE Trans. Elec. Dev., 61, p.3081 (2014).

[18] M. Duan, J. F. Zhang, A. Manut, Z. Ji, W. Zhang, A. Asenov, L. Gerrer, D. Reid, H. Razaidi, D. Vigar, V. Chandra, R. Aitken, B. Kaczer, and G. Groeseneken iedm, p.547, 2015.

[19] M. Duan, J. F. Zhang, Z. Ji, W. Zhang, D. Vigar, A. Asenov, L. Gerrer, V. Chandra, R. Aitken, and B. Kaczer, IEEE Trans. Elec, Dev., 63, p. 3642 (2016).

[20] M. Duan, J. F. Zhang, Z. Ji, W. Zhang, B. Kaczer, and A. Asenov, IEEE Trans. Elec, Dev., 64, p.2478 (2017).

[21] M. Duan et al., IEDM, p. 774 (2013). 